\title{
On the electrical breakdown of gaseous dielectrics-an engineering approach
}

\author{
Pedersen, Aage
}

Published in:

Conference on Electrical Insulation and Dielectric Phenomena

Link to article, DOI:

10.1109/CEIDP.1989.69523

Publication date:

1989

Document Version

Publisher's PDF, also known as Version of record

Link back to DTU Orbit

Citation $(A P A)$ :

Pedersen, A. (1989). On the electrical breakdown of gaseous dielectrics-an engineering approach. In Conference on Electrical Insulation and Dielectric Phenomena (pp. 21-58). IEEE.

https://doi.org/10.1109/CEIDP.1989.69523

\section{General rights}

Copyright and moral rights for the publications made accessible in the public portal are retained by the authors and/or other copyright owners and it is a condition of accessing publications that users recognise and abide by the legal requirements associated with these rights.

- Users may download and print one copy of any publication from the public portal for the purpose of private study or research.

- You may not further distribute the material or use it for any profit-making activity or commercial gain

- You may freely distribute the URL identifying the publication in the public portal

If you believe that this document breaches copyright please contact us providing details, and we will remove access to the work immediately and investigate your claim. 


\section{ON THE ELECTRICAL BREAKDOWN OF GASEOUS DIELECTRICS AN ENGINEERING APPROACH}

A. Pedersen, Fellow IEEE

Physics Laboratory II, Building 309B

The Technical University, DK-2800 Lyngby, Denmark

\section{INTRODUCTION}

A knowledge of spark breakdown for various gap geometries is essential when designing gas-insulated high-voltage apparatus. A large amount of experimental data is available in standard textbooks on high voltage engineering and electrical breakdown of gases. Many empirical formulae are available from which breakdown or corona onset field strengths may be calculated. Such formulae are, however, valid only within certain ranges, and extrapolation can lead to large errors. It may, therefore, be of interest to consider the possibilities for a direct application of the physics of gaseous breakdown in the design of gas-insulated high-voltage equipment.

The classical Townsend theory of the growth of ionization is fundamental to any discussion of breakdown of gaseous dielectrics. It is generally accepted that the Townsend theory of breakdown can account for the onset of breakdown in uniform fields under quasi-static conditions. However, in order to give a detailed description of the many observed phenomena of engineering importance, the Townsend theory must be supplemented by the streamer concept of breakdown. Based on these physical concepts quantitative criteria for the onset of breakdown have been proposed. These are, however, of limited value to the high voltage engineer. The main problem is that such criteria are difficult to apply to practical situations. The necessary physical data may either not be available or, if so, are presented in a form which is alien to engineering applications.

Another complication is related to the fact that the growth of ionization is exponential. This can, owing to the mathematical nature of exponential functions, lead to procedures which appear to give acceptable results. A 
closer analysis may reveal, however, that quite meaningless properties are hidden in the criteria. For example, the electron avalanche may contain an impossibly large number of electrons, but the criteria can still yield acceptable breakdown data. It is in a way too easy to formulate a criterion for breakdown. The effects of these mathematical difficulties can be minimized, however, by formulating the quantitative criterion in such a way that it does not contain any parameter which is chosen arbitrarily and thus acts as a sort of "fiddle factor". An example of such a parameter can be the $K$-factor in the streamer criterion for breakdown. A consistent and physically meaningful approach to the evaluation of $K$ is adopted in the present paper.

Following a discussion of the basic concepts, quantitative criteria will be derived for the onset of breakdown in atmospheric air and similar weakly-electronegative gases. Thereafter, a comparable criterion is formulated for strongly-electronegative gases such as $\mathbf{S F}_{6}$. These criteria contain no quantities other than those which can be obtained directly from breakdown voltage measurements in a uniform field, i.e. from Paschen curve data. No specific data are required for the ionizing coefficients of the gas. The advantage, from an engineering point of view, is that it is much easier to perform reliable Paschen curve measurements than it is to measure ionization growth parameters. The price payed is that criteria formulated in this way cannot be employed to yield unknown Paschen curves.

The criteria can be applied to any nonuniform field configuration provided the electrostatic field distribution is known. Moreover, in many cases, a simple analytical approximation to the field distribution can render an extensive calculation of the electrostatic field unnecessary.

The following discussions will deal exclusively with situations in which the onset of breakdown in the system is synonymous with the onset of a corona discharge. Breakdown situations in which corona stabilization is operative are not addressed as these are of limited practical relevance with respect to the design of electrical insulation. 


\section{BASIC CONCEPTS}

\section{The Current in the Lead}

The currents flowing in the gas between the electrodes during the formation of a breakdown are related to the motion of electrons and ions in the applied electric field. These spatially distributed currents can be described by the current density $\vec{J}$ given by

$$
\vec{J}=\sum_{v=0}^{n} \rho_{v} \vec{u}_{v}
$$

where $\rho$ is the charge density and $\vec{u}$ the drift velocity, $v=0$ refers to electrons and $\mathrm{n}$ is the number of possible species of positive and negative ions. The relationship between these distributed currents and the current flowing in the lead to an electrode often causes conceptual difficulties.

Let I denote the current flowing in the lead towards the electrode and let $I_{t}$ be the current which represents the transfer of charge from the electrode into the interelectrode space. These currents are, as a consequence of the principle of the conservation of charges, related to the net charge $Q$ on the electrode in the following way

$$
I=I_{\mathrm{t}}+\frac{\mathrm{d} Q}{\mathrm{~d} t} \text {. }
$$

The charge $Q$ would, in the absence of inter-electrode space-charges, simply be given by the capacitance $C$ of the system and the applied voltage $U$, i.e. $Q=C U$. In the presence of inter-electrode space-charges, an additional charge, the induced charge $q$, will however occur on the electrode. The net charge thus becomes

$$
Q=q+C U \text {. }
$$

The induced-charge depends in a unique way on the magnitudes and locations of the inter-electrode space-charges, but it is independent of the applied voltage. An infinitesimal charge d $Q$ somewhere between the electrodes will induce a charge $\mathrm{d} q$ on the electrode and $\mathrm{d} q$ will, in view of the principle of superposition, be proportional to dQ, i.e. 


$$
\mathrm{d} q=-\varphi \mathrm{d} Q
$$

The dimensionless quantity $\varphi$ is a positive scalar function which depends on the location of $d Q$ only. The entire induced-charge on the electrode will thus be given by

$$
q=-\iiint \varphi \rho \mathrm{d} \Omega
$$

in which $\rho$ is the volume-charge density at the location of the volume element $d \Omega$. The volume integral is extended over all the space between the electrodes.

In the absence of polarizable material the function $\varphi$ is, as shown by Maxwell [1], a solution to Laplace's equation

$$
\nabla^{2} \varphi=0
$$

with the boundary conditions $\varphi=1$ at the surface of the measuring electrode and $\varphi=0$ at all other electrodes. Any available method of electrostatic field calculation can be used to evaluate $\varphi$. Let $V_{c}$ be the potential at a point in the inter-electrode space in the absence of any space-charges when the potential of the electrode is $U_{c}$ and all other electrodes are at zero potential. $V_{c}$ is then given by

$$
\nabla^{2} v_{c}=0
$$

This means that

$$
\varphi=V_{c} / U_{c}
$$

It is important to note that $V_{c}$ and $U_{c}$ are entirely fictitious quantities such that $U_{c}$ can be given any arbitrarily chosen value, i.e. $U_{c}$ is not synonymous with the applied voltage during the formation of a gaseous discharge. For this reason the expression $V_{c} / U_{c}$ should not be inserted in equation (5).

In the presence of polarizable materials, e.g. when discussing gaseous breakdown within voids in solid dielectrics, the function $\varphi$ must be replaced by another dimensionless function $\lambda[2,3,4]$. This function is also given by Laplace's equation but in the following form

$$
\operatorname{div}(\epsilon \operatorname{grad} \lambda)=0,
$$


in which $\epsilon$ is the permittivity of the polarizable dielectric. The boundary conditions are $\lambda=1$ at the surface of the electrode and $\lambda=0$ at all other electrodes. In addition, the following condition must be fulfilled at all dielectric interfaces

$$
\epsilon_{+}(\partial \lambda / \partial n)_{+}=\epsilon_{-}(\partial \lambda / \partial n)_{-}
$$

where the signs + and - refer to the two sides of the interface and $\lambda$ is differentiated in the direction normal to the interface. Any standard method for calculation of space-charge-free electrostatic fields can be applied to evaluate $\lambda$ from the equations

$$
\begin{gathered}
\operatorname{div}\left(\epsilon \operatorname{grad} V_{c}\right)=0, \\
\epsilon_{+}\left(\partial V_{c} / \partial n\right)_{+}=\epsilon_{-}\left(\partial V_{c} / \partial n\right)_{-}
\end{gathered}
$$

and

$$
\lambda=V_{\mathrm{c}} / U_{\mathrm{c}} .
$$

$U_{C}$ is the arbitrarily chosen potential of the electrode and $V_{C}$ is the potential of a point in the inter-electrode space. All other electrodes are at zero potential.

The current flowing in the lead towards the electrode is

$$
I=I_{\mathrm{t}}+\frac{\mathrm{d} q}{\mathrm{~d} t}+C \frac{\mathrm{d} U}{\mathrm{~d} t} .
$$

Differentiation of equation (5) with respect to the time $t$ yields

$$
\frac{\mathrm{d} q}{\mathrm{~d} t}=-\iiint \varphi \frac{\partial \rho}{\partial t} \mathrm{~d} \Omega
$$

which by means of the continuity equation

$$
\operatorname{div} \vec{J}+\frac{\partial \rho}{\partial t}=0
$$

can be written in the form

$$
\frac{\mathrm{d} q}{\mathrm{~d} t}=\iiint \varphi \operatorname{div} \vec{J} \mathrm{~d} \Omega
$$

or

$$
\frac{\mathrm{d} q}{\mathrm{~d} t}=\iiint \operatorname{div}(\overrightarrow{\varphi \vec{J}}) \mathrm{d} \Omega-\iiint \vec{J} \cdot \operatorname{grad} \varphi \mathrm{d} \Omega
$$

Application of the divergence theorem of Gauss to the 
first volume integral shows, since $\varphi=1$ at the electrode, that

$$
I_{t}+\iiint \operatorname{div}(\varphi \vec{J}) \mathrm{d} \Omega=0 .
$$

Insertion in equations (18) and (14) gives the following expression for the current $I$ flowing in the lead towards the electrode when charges are in motion in the interelectrode space

$$
I=-\iiint \vec{J} \cdot \operatorname{grad} \varphi \mathrm{d} \Omega+c \frac{\mathrm{d} U}{\mathrm{~d} t} .
$$

It should be noted that $I_{t}$, i.e. the current representing the actual transfer of charge from the electrode to the gap, does not appear explicitly in the expression for the current in the lead. The reason is that this transfer of charge is associated with a corresponding simultaneous change in the induced-charge without any transfer of charge through the lead to the electrode. The charges which are transferred to the gap from the electrode contribute to the current in the lead solely by the effects of their motions in the gap between the electrodes.

Since $\varphi$ is a solution to Laplace's equation, see equations ( 6$),(7)$ and ( 8 ), the gradient of $\varphi$ can be found from

$$
\operatorname{grad} \varphi=-\vec{E}_{\mathrm{C}} / U_{\mathrm{C}}
$$

in which $\vec{E}_{C}$ is the field strength in the space-charge free electrostatic field between the electrodes when the electrode is given the arbitrarily chosen potential $U_{c}$ and all other electrodes are at zero potential. It must be emphasized that $U_{C}$ is not synonymous with the applied voltage during discharge activities. Insertion of equation (21) in equation (20) should therefore not be made.

Formulae similar to equation (20) have been given by many authors, see for example von Engel and Steenbeck [5], Shockley [6], and Ramo [7]. These formulae are referred to by some authors as the Ramo-shockley theorem. It should, however, be remembered that quantitative treatments of this problem based on the concept of induced charges date back at least to Maxwell. This will be found in the first edition of his Treatise [1], but not in the third edition edited by J.J. Thomson. 


\section{The Effective Coefficient of Ionization}

Growth of ionization can be approached in two different ways. From a molecular point of view, it is the individual ionizing collision processes described in terms of probabilities and collision cross-sections which are of primary interest. In high voltage engineering, it is the collapse of the voltage across a gap as a result of gaseous breakdown which is of paramount importance. This phenomenon involves so many collision processes that it is natural to consider the event as a macroscopic phenomenon described in terms of macroscopic swarm parameters such as primary and secondary coefficients of ionization. The number of electrons participating in the ionizing processes is then so large that, in view of the Law of Large Numbers [8], it makes sense to represent the actual number of electrons, which of course can take only integer values, by a continuous function. This makes it possible to apply mathematical concepts such as differentiation and integration to the analysis of the growth of ionization in electron avalanches.

From a pragmatic point of view, the effective coefficient of ionization $\bar{\alpha}$ is defined by means of the differential equation

$$
\mathrm{d} N(z)=\bar{\alpha}(z) N(z) \mathrm{d} z,
$$

with $z$ being a coordinate along the field line in the electric field along which an electron avalanche is developing. $N(z)$ is a continuous, differentiable function which represents the actual number of electrons $N_{e}$ participating in the collision processes.

Equation (22) has no physical sense unless swarm conditions exist; i.e. $N_{e}$ must be sufficiently large such that $N_{\mathrm{e}}$ can be represented by $N(z)$. Moreover, $N_{\mathrm{e}}$ must be so large that the Law of Large Numbers is operative. This ensures that, for a given value of $N(z)$, proportionality exists between $\mathrm{d} N(z)$ and $\mathrm{d} z$. If $N_{\mathrm{e}}$ is so small that the Law of Large Numbers is inoperative the statistical nature of the individual collision processes must be taken into account. Application of equation (22) to a single electron or to a few electrons has no meaning. The conventional written definition of $\bar{\alpha}$ in terms of number of ionizing collisions per electron per unit length in the 
direction of the field is, therefore, at variance with the proper mathematical definition.

From a mathematical point of view, swarm conditions imply that $\bar{\alpha}$ is defined for a continuum, and that $\mathrm{d} N(z)$ is a differential which represents the actual number of electrons $\Delta N_{e}$ produced by $N_{e}$ electrons drifting a distance $\Delta z$ in the direction of the electric field. Hence $\bar{\alpha}$ is essentially a coefficient of proportionality.

Although $\bar{\alpha}$ results from a statistical average of a large number of collision processes, $\bar{a}$ itself is not a statistical variable. It is a macroscopic quantity similar in nature to, for example, the temperature or the pressure of a gas.

\section{Electron Avalanche Development}

The application of equation (22) to the growth of an electron avalanche is normally restricted to the later stages of the development. The reason is that initially $N_{\mathrm{e}}$ will be so small that swarm conditions do not exist. An important consequence of this limitation is that the growth of an electron avalanche will initially not be exponential. The exponential growth will begin when the number of electrons attains such a value that swarm conditions exist. Let $z_{1}$ denote the lowest value of $z$ for which $N_{e}$ is so large that swarm conditions exist, then the development of the avalanche for $z>z_{1}$ will be exponential in these circumstances and given by

$$
N(z)=N\left(z_{1}\right) \exp \int_{z_{1}}^{z} \bar{\alpha}\left(z^{\prime}\right) \mathrm{d} z^{\prime} .
$$

$N\left(z_{1}\right)$ represents the minimum number of electrons for which equation (22) is valid. It should be noted that the values of both $N\left(z_{1}\right)$ and $z_{1}$ are unknown and variable. The transition from discrete behaviour to swarm conditions is moreover likely to be gradual. This means that specific values for $z_{1}$ and $N\left(z_{1}\right)$ cannot be given for the growth of an electron avalanche in a given electric field. Evidence for this variation is clearly provided by the scatter observed in the magnitude of single avalanche current pulses recorded under constant experimental conditions; see Raether [9].

It should be emphasized that the application of the dif- 
ferential equation (22) to the development of an avalanche implies that this part of the growth is, in principle, deterministic; i.e. if $N\left(z_{1}\right)$ and $z_{1}$ were known, $N(z)$ would be given exactly by equation (23). In general, however, $z_{1}$ and $N\left(z_{1}\right)$ will not be known, and hence the actual magnitude of $N(z)$ can never be predicted.

\section{The Onset Breakdown Voltage}

The formation of a spark breakdown requires that at least one electron is present at a suitable place in the gap when the voltage is applied. These requirements introduce a statistical time-lag $t_{s}$ in the formation of a spark. This time-lag is of particular importance if the applied voltage increases in time, since finite values of $t_{\mathrm{s}}$ will lead to higher breakdown voltages. $t_{s}=0$ implies that, with respect to the instantaneous value of the applied voltage, the initiatory electron is in the correct spatial location, such that a minimum voltage is required to break down the gap. Such conditions are most readily met in near-uniform field geometries under the application of a highly stabilized d.c. voltage, together with suitable irradiation of the cathode surface. This minimum breakdown voltage is called the onset breakdown voltage. Precision breakdown voltage measurements in $\mathrm{SF}_{6}$ have indicated that the standard deviation in a series of measurements of the onset breakdown voltage can be as low as 3 volts in 62000 volts [10]. It is thus an extremely well defined quantity.

A knowledge of the onset breakdown voltage is of paramount importance in the design of gas insulated systems, and it is a necessity when the reliability of such systems is under consideration. Since the precise physical mechanisms of spark breakdown are as yet not fully understood, all existing criteria for the prediction of onset breakdown voltages are essentially empirical. However, because of the dominating influence of the exponential growth term in the mathematical expression for the development of an electron avalanche, all such criteria $[9,11,12,13]$ can in effect be reduced to the same mathematical expression, viz.

$$
\int_{0}^{z_{0}} \bar{a}(z) d z=K \text {, }
$$


in which $z$ is a distance coordinate along the field line in question, $z_{0}$ is the critical avalanche length and $K$ is a dimensionless parameter. This simple expression was originally proposed by Schumann $[14,15]$ but is today normally referred to as the streamer breakdown criterion.

\section{CRITERIA FOR BRFAKDOTN}

\section{The Townsend Criterion}

The Townsend concept of breakdown is associated with the transition from a steady-state gas amplified current to a self-sustained discharge $[16,17,18]$. When this concept was conceived, the main interest was in low pressure discharges for which a discussion in terms of uniform fields was adequate. The Townsend breakdown criterion was therefore formulated with special reference to uniform fields and to situations which could be described as one-dimensional. However, with respect to high voltage insulation problems of today the nonuniform field situation at high gas pressures is of particular importance.

Let us consider a nonuniform field between two electrodes. One of the electrodes, the cathode, is irradiated such that a steady current $I$ is flowing in the external circuit when a d.c. voltage is applied, i.e. the number of electrons emitted from the cathode per area and time is so large that swarm conditions exist. In the absence of ionizing processes in the gas the current in the lead will be given by

$$
I=\iint \vec{J}_{0} \cdot \vec{e} \mathrm{~d} S
$$

The surface integral is extended over the surface of one of the electrodes. $\vec{J}_{0}$ denotes the current density at the surface element $d S$ and $\vec{e}$ is a unit vector normal to and directed away from the electrode.

The current in the external circuit will attain a higher value if the applied voltage is increased to a level at which ionizing processes are active in the gas. The effect upon the current of these ionizing processes can be taken into account by expressing the current in the following form

$$
I=\iint G \vec{J}_{0} \cdot \vec{e} \mathrm{~d} S
$$


in which $G$ is a dimensionless scalar function. The value of $G$ depends on the location of $\mathrm{d} S$.

The current in such an externally maintained prebreakdown Townsend discharge will normally be so low that it is justified to neglect the effect of the charge carriers on the electrostatic field distribution. This means that the flow of the electrons and ions will follow the field lines in the space-charge-free Laplacian field between the electrodes. The contribution $d I$ to the current which is associated with the surface element $\mathrm{d} S$ will thus flow in a Faraday field tube defined by the contour of $\mathrm{d} S$. Since the cross-section of such a field tube is infinitesimal we can consider the development of $d I$ to be onedimensional.

An expression for $G$ in terms of the primary and secondary ionizing parameters can be derived in the following way. Let us consider the ionizing processes associated with $N_{0}$ electrons which are emitted from the cathode within the surface element d $S . N_{0}$ is so large that swarm conditions exists. The total number of negative charge carriers, i.e. electrons and negative ions, which, in the absence of secondary processes, will arrive at the anode will be $N_{0}$ plus a number which is equal to the total number of ionizing collisions within the Faraday field tube. The number of primary ionizing collisions between $z$ and $z+\mathrm{d} z$ is

$$
\mathrm{d} n_{1}(z)=\alpha(z) N(z) \mathrm{d} z
$$

in which $\alpha$ is Townsend's first coefficient of ionization and

$$
N(z)=N_{0} \exp \int_{0}^{z} \bar{\alpha}\left(z^{\prime}\right) \mathrm{d} z^{\prime}
$$

is representing the number of electrons at $z$. The total number of ionizing collisions within the field tube thus becomes

$$
n_{1}(\ell)=N_{0} g(\ell)
$$

with

$$
g(\ell)=\int_{0}^{\ell} \alpha(z)\left[\exp \int_{0}^{z} \bar{\alpha}\left(z^{\prime}\right) \mathrm{d} z^{\prime}\right] \mathrm{d} z,
$$

where $\ell$ is the length of a field line within the field tube. If secondary ionizing processes are active an addi- 
tional number of electrons will be emitted from the cathode within the surface element ds. Let $\mathrm{dn}_{2}(z)$ denote the number of such secondary electrons leaving the cathode due to collision processes between $z$ and $z+d z$, then

$$
d n_{2}(z)=\omega(z) N(z) d z
$$

in which $\omega(z)$ is a generalized secondary coefficient of ionization, see $[17,18]$. Integration of equation (31) yields

$$
n_{2}(\ell)=N_{0} h(\ell)
$$

with

$$
h(\ell)=\int_{0}^{\ell} \omega(z)\left[\exp \int_{0}^{z} \bar{\alpha}\left(z^{\prime}\right) \mathrm{d} z^{\prime}\right] \mathrm{d} z
$$

in which $n_{2}(\ell)$ is the total number of the first generation of secondary electrons emitted from the cathode within the surface element $d S$. It is evident that secondary electrons are related directly to collision processes within the field tube defined by d $S$. Additional secondary electrons will be liberated within d $S$ due to collision processes in adjacent flux tubes. These extra secondary electrons are taken into account by including them in $N_{0}$. The total number $N(\ell)$ of negative charge carriers which eventually will reach the anode as a result of the emission of $N_{0}$ electrons within the surface element $\mathrm{d} S$ thus becomes

$$
\begin{aligned}
N(\ell)= & N_{0}[1+g(\ell)]+N_{0}[1+g(\ell)] h(\ell)+ \\
& N_{0}[1+g(\ell)][h(\ell)]^{2}+\ldots
\end{aligned}
$$

This series will converge if $h(\ell)<1$, and we obtain

$$
N(\ell)=N_{0} \frac{1+g(\ell)}{1-h(l)} \text {. }
$$

The function $G$ in equation (26) is thus given by

$$
G=\frac{1+\int_{0}^{\ell} \alpha(z)\left[\exp \int_{0}^{z} \bar{\alpha}\left(z^{\prime}\right) \mathrm{d} z^{\prime}\right] \mathrm{d} z}{1-\int_{0}^{\ell} \omega(z)\left[\exp \int_{0}^{z} \bar{\alpha}\left(z^{\prime}\right) \mathrm{d} z^{\prime}\right] \mathrm{d} z}
$$

In a uniform field $\alpha, \bar{\alpha}$, and $\omega$ will be constants and $\ell$ will be equal to the gap length $d$. Consequently equation 
(35) simplifies, and following this equation (26) leads to the well-known uniform field formula

$$
I=I_{0} \frac{1+(\alpha / \bar{\alpha})[\exp (\bar{\alpha} d)-1]}{1-(\omega / \bar{\alpha})[\exp (\bar{\alpha} d)-1]} .
$$

The series given by equation (33) becomes divergent if $h(l)$ is equal to or greater than one. The Townsend criterion for the onset of breakdown in a nonuniform field is thus given by

$$
\int_{0}^{\ell} \omega(z)\left[\exp \int_{0}^{z} \bar{\alpha}\left(z^{\prime}\right) \mathrm{d} z^{\prime}\right] \mathrm{d} z=1 .
$$

A direct application of the Townsend criterion to realistic electrode arrangements requires a knowledge of the parameters $\bar{\alpha}$ and $\omega$. Whereas $\bar{\alpha}$ is relatively well documented for many gases of engineering importance, meaningful information on $\omega$ is hardly available. The reason is that $\omega$ depends not only on field strength and gas pressure, but also on the overall geometry of the system.

In an attempt to overcome these difficulties, schumann [14] noted that the Townsend criterion would imply a polarity effect in the onset breakdown voltage if it is applied to a gap geometry for which the field distribution is not only nonuniform but also asymmetric. The field along the axis of the standard sphere-gap with one sphere earthed is such a nonuniform and asymmetric field distribution. If the applied voltage is of positive polarity the earthed sphere will be the cathode, and the direction of the integration in the Townsend criterion is then from the earthed sphere towards the high voltage sphere, whereas the direction of the integration is reversed for negative polarity. This will, because of the mathematical structure of the Townsend criterion, lead to a polarity effect in the d.c. onset breakdown voltage. Schumann noted that such a polarity effect is absent [19], and that this effect disappears from the Townsend criterion if one assumes that $\omega(z)$ is proportional to $\bar{\alpha}(z)$ [14]. The insertion of $\omega(z)=k \bar{\alpha}(z)$, where $k$ is a constant, in the Townsend criterion leads to

$$
\exp \int^{\ell} \bar{\alpha}(z) \mathrm{d} z=\frac{k+1}{k}
$$

or to the simple form known as the Schumann criterion 


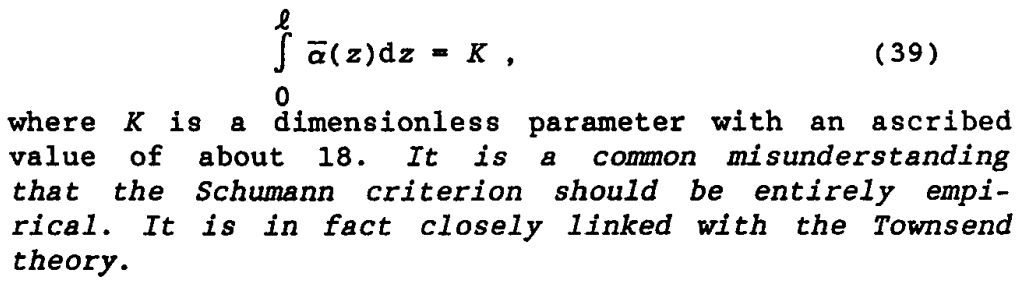

where $K$ is a dimensionless parameter with an ascribed value of about 18 . It is a common misunderstanding that the Schumann criterion should be entirely empirical. It is in fact closely linked with the Townsend theory.

\section{The Streamer Criterion}

Whereas the Townsend theory of spark breakdown depends on many generations of avalanches, a single electron avalanche mechanism is the fundamental characteristic of the streamer concept of breakdown $[9,20]$. It is, however, likely that more than one avalanche are in fact required in order to create the background for the growth of the avalanche which eventually precipitates the formation of a spark channel [21]. Field distortion and photo-ionization in the gas have been considered as possible active mechanisms. A proper physical theory for the streamer concept of breakdown has, however, never been formulated. In spite of this situation it is nevertheless possible, by utilizing the inherent mathematical nature of exponential growth, to formulate a quantitative criterion from which the onset value of the breakdown voltage can be calculated if the electric field distribution is known $[22,23]$.

It is tacitly assumed that breakdown occurs when the number of electrons $N_{\mathrm{e}}$ in an electron avalanche reaches a critical value $N_{\mathrm{c}}$. The actual value of $N_{\mathrm{C}}$ is not known, but it is assumed to be of the order of $10^{8}$ irrespective of the specific conditions under consideration.

The growth of an avalanche initiated at $z=0$ by one or more starting electrons will, as discussed earlier, not be exponential until $z$ has reached a value $z_{1}$ where the number of electrons $N\left(z_{1}\right)$ is so large that swarm conditions exist. Thereafter the growth of the avalanche will be controlled by equation (23).

The length of the avalanche when it attains the critical size $N_{C}$ is called the critical avalanche length $z_{0}$. In a uniform field, or a weakly nonuniform field, $z_{0}$ will be synonymous with the gap length $d$. In a nonuniform field 
$z_{0}$ is the distance from the electrode along the field line in question to the point beyond which the growth of the avalanche ceases, i.e. to the point at which $\bar{\alpha}=0$.

Owing to the random nature of the initial stages of the avalanche growth, i.e. for $z<z_{1}$, the value of $z_{1}$ will vary considerably for a series of single avalanches developing along the same path. Consequently, for any value of $z>z_{1}$, a maximum value, $N(z)_{\max }$, for $N(z)$ will be obtained for a minimum value of $z_{1}$. Therefore, for a finite avalanche length, the onset of breakdown will be associated with the avalanche possessing the largest number of electrons.

The criterion for the onset of breakdown can thus be expressed in the following form

$$
N_{\mathrm{C}}=N\left(z_{0}\right)_{\max }
$$

with

$$
N\left(z_{0}\right)=N\left(z_{1}\right) \exp \int_{z_{1}}^{z_{0}} \bar{\alpha}(z) \mathrm{d} z .
$$

The criterion can be re-written in the following manner

$$
N_{\mathrm{C}}=\left[\frac{N\left(z_{1}\right) \exp \int_{0}^{z_{0}} \bar{\alpha}(z) \mathrm{d} z}{\exp \int_{0}^{z_{1}} \bar{\alpha}(z) \mathrm{d} z}\right]_{\max } .
$$

This procedure is valid mathematically. It is, however, important to realize that, whereas the exponential term in equation (41) has a meaningful physical interpretation, this is not the case for the two exponential terms in equation (42) when considered individually. They do of course have a mathematical meaning since $\bar{\alpha}$ is a continuous function of the electric field strength and thus of $z$.

The variation in the size of the electron avalanches means that the value of $N\left(z_{1}\right)$ can differ considerably from the value of the exponential term in the denominator of equation (42). In addition, at the onset of breakdown, the numerical value of their ratio will have attained a maximum, corresponding to a minimum value for $z_{1}$. It is, 
therefore, evident from equation (42) that at the onset of breakdown

$$
\begin{aligned}
& N_{\mathrm{c}} \neq \exp \int_{0}^{z_{0}} \bar{\alpha}(z) \mathrm{d} z \\
& \text { i.e. } \quad \ln N_{\mathrm{c}}+\int_{0}^{z_{0}} \bar{\alpha}(z) \mathrm{d} z .
\end{aligned}
$$

The true size of the electron avalanche at the onset of breakdown is consequently not given by the integral in (44), an assertion which is frequently seen in the literature. Nevertheless, because of the exponential nature of the variation of $\bar{\alpha}$ with $E$, this integral will be so dominant a parameter in equation (42) that it will effectively control the onset condition for breakdown, such that breakdown will occur when this integral attains a certain value $K$. Thus the streamer criterion can be written as

$$
\int_{0}^{z_{0}} \vec{\alpha}(z) d z=K .
$$

It is evident that this criterion is strictly empirical.

In the literature $K$ is often given the value 18; this being the natural logarithm of the value generally assumed for $N_{\mathrm{C}}$, viz, $10^{8}$. However, since it follows from (44) that

$$
K \neq \ln N_{\mathrm{C}}
$$

there is no reason apart from tradition for retaining $K$ equal to 18 .

So far it has been tacitly assumed that the electric field $E(z)$ varies monotonically with $z$ between zero and $z_{0}$, such that the integral of $\bar{\alpha}$ would also be a monotonically varying function of $z$. In general such a variation will be encountered. However, in some situations $E(z)$ will not vary monotonically within the interval $0<z$ $<z_{0}$. An example of this is the axial field between two spheres for which the ratio of gap length $d$ to sphere diameter $D$ is such that $z_{0}=d$. In such situations the application of the streamer criterion is valid if, for all values of $z$ in the interval $z_{1}<z<z_{0}$.

$$
\int_{z_{1}}^{z} \bar{\alpha}\left(z^{\prime}\right) \mathrm{d} z^{\prime} \geqq 0 \text {. }
$$


since this implies that $N(z)$ will never be less than $N\left(z_{1}\right)$, thus ensuring swarm conditions. However, since the value of $z_{1}$ is unknown, (47) is with reference to numerical calculations of little practical value. As a substitute the less rigorous condition

$$
\int_{0}^{z} \bar{\alpha}\left(z^{\prime}\right) \mathrm{d} z^{\prime}>0,
$$

can be used as an indication for the validity of any calculations. It should, however, be noted that fulfillment of (48) does not automatically imply swarm conditions.

\section{ENGINEERING APPLICATIONS}

\section{Field Distribution along a Field Line}

A knowledge of the electrostatic field is a necessity for an application of the breakdown criterion. Many methods are available from which the required information can be obtained. However, since we are interested only in the field along certain field lines, most standard procedures lead to an excess of data. Whereas the entire field is a solution to a second-order partial differential equation, i.e. Laplace's equation, the field distribution along a field line is given by a first-order ordinary differential equation.

In a Laplacian field the field strength $E$ at a given point $P$ in space and the mean curvature $H$ of the equipotential surface through $P$ are related by the first-order ordinary differential equation, Green's differential equation, namely

$$
\frac{\mathrm{d} E(z)}{\mathrm{d} z}+2 H(z) E(z)=0
$$

in which $z$ is a coordinate along the field line through $P$. For a regular surface the mean curvature $H$ is defined by

$$
H=\frac{1}{2}\left(\frac{1}{R_{1}}+\frac{1}{R_{2}}\right) \text {, }
$$

where $R_{1}$ and $R_{2}$ are the radii of curvature of the curves through $P$ of the normal sections of two mutually perpendicular planes. In practice $R_{1}$ and $R_{2}$ are normally taken to be the two principal radii of curvature, i.e. the maximum and the minimum values of all the possible sets 
of $R_{1}$ and $R_{2}$. It should be noted that the sum of $1 / R_{1}$ and $1 / R_{2}$ is constant as long as these are referred to two mutually perpendicular planes, see [24].

Although expressed in terms of potential, equation (49) was first derived by Green in his famous Essay on potential theory [25]. Since then, equation (49) has been rederived several times, see $[26,27]$. Integrating Green's equation we obtain the following general expression

$$
E(z)=E(0) \exp \left[-2 \int_{0}^{z} H\left(z^{\prime}\right) \mathrm{d} z^{\prime}\right] \text {. }
$$

where $z$ ' is a dummy variable, see [28]. An exact application of this expression would require a knowledge of $H(z)$. It is possible, however, by means of suitable approximations for the unknown $H(z)$ to utilize equation (51) for breakdown onset calculations. For example, if we are primarily interested in the field along a field line in the proximity of a high voltage conductor, we may consider $H(z)$ to be constant. A first approximation to equation (51) for small values of $z$ will then be

$$
E(z)=E(0)[1-2 H(0) z] \text {, }
$$

in which $z$ is the distance along the field line from the surface of the electrode. $E(0)$ and $H(0)$ are the values of field strength and mean curvature at the location of the field line on the electrode. The range of validity of equation (52) can be estimated by examining the fields of two simple geometries, namely the isolated sphere and the isolated circular cylinder. These are the simplest geometries which encompass the overall $\left(R_{1}, R_{2}\right)$ variation of interest, i.e.

(a) $R_{1}$ finite, $R_{2}$ finite and $R_{1}=R_{2}$;

(b) $R_{1}$ finite, $R_{2}$ infinite.

For these fields, the associated variations of $H(z)$ are, on the basis of equation (50),

$$
H(z)=\frac{H(0)}{1+H(0) z}
$$

for the isolated sphere, and

$$
H(z)=\frac{H(0)}{1+2 H(0) z}
$$


for the isolated circular cylinder. Upon insertion in equation (51) and integration, we obtain expressions for the two field distributions in terms of conductor mean curvature. For the isolated sphere we obtain

$$
E(z)=\frac{E(0)}{[1+H(0) z]^{2}}
$$

and for the isolated circular cylinder

$$
E(z)=\frac{E(0)}{1+2 H(0) z} .
$$

For $H(0) z<1$, these exact expressions can be expanded in terms of $H(0) z$. Thus for the isolated sphere we obtain

$E(z) / E(0)=1-2 H(0) z+3[H(0) z]^{2}-4[H(0) z]^{3}+\ldots$

and for the isolated circular cylinder

$E(z) / E(0)=1-2 H(0) z+4[H(0) z]^{2}-8[H(0) z]^{3}+\ldots$

from which it is seen that, for $H(0) z<0.1$, equation (52) represents a rather good appoximation to the field near a high voltage electrode.

An important area of application of Green's differential equation (49) is in the assessment of numerical field calculations. As a knowledge of the conductor geometries is a prerequisite of any numerical method of solving Laplace's equation, the determination of the mean curvature of the different conductors is independent of the field calculations. Consequently the fulfilment of the differential equation (49) at a conductor surface can be used to check the accuracy of the numerical field calculation. If the conductor as a whole is considered to be associated with $z=0$, then for the selected location we compare the value obtained for

$$
-\frac{1}{E(0)}\left[\frac{\mathrm{d} E(z)}{\mathrm{d} z}\right]_{z=0}
$$

with the corresponding value of $2 H(0)$. This method of checking is an intrinsic feature of the charge simulation method of field calculation developed by steinbigler [29].

The field distribution in the proximity of a conductor 
surface can be checked by means of equation (52). For example, if the field distribution $E(z) / E(0)$ is plotted as a function of the normalized distance $H(0) z$ along the field line, then the gradient of the resulting graph at $H(0) z=0$ will be independent of both $H(0)$ and $E(0)$ and have a value of -2 for convex surfaces. This characteristic gradient of -2 can be used as a checking factor.

Apart from concave surfaces which will yield a gradient of +2 , the only exception to the general rule is a plane surface for which $H(0)=0$. The gradient should thus be zero at the conductor surface. Other surfaces can also display $H(0)=0$. However, since this implies that $R_{1}=-R_{2}$ such locations are invariably associated with low field regions and are therefore of little interest in gas breakdown studies.

Breakdown in Atmospheric Air

No other gas is so widely used as an insulating medium as atmospheric air. Since the dielectric properties depend on temperature, pressure and humidity it is customary to refer to a set of standard conditions. These are $20{ }^{\circ} \mathrm{C}$, $1013.25 \mathrm{hPa}$, and a water vapour content of $11 \mathrm{~g}$ per $\mathrm{m}^{3}$. However, since air is also used as an insulating gas at higher pressures it is convenient to use $1000 \mathrm{hPa}=1 \mathrm{bar}$ as the reference pressure. In view of the dimensions actually encountered in high voltage design work, field strengths will be quoted in $\mathrm{kV} / \mathrm{mm}$ rather than $\mathrm{MV} / \mathrm{m}$ although these units are identical. Similarly $E / P$, where $p$ is the gas pressure, will be given with the unit $\mathrm{kV}$ (mm bar $)^{-1}$; we have $1 \mathrm{kV}(\mathrm{mm} \text { bar })^{-1}=10 \mathrm{~V}(\mathrm{~m} \mathrm{~Pa})^{-1}$.

From a practical point of view the only realistic approach to breakdown voltage calculations is the application of the empirical criterion

$$
\int_{0}^{z_{0}} \bar{\alpha}(z) \mathrm{d} z=K .
$$

Data for $\bar{\alpha} / p$ as a function of $E / p$ for atmospheric air are available in the literature $[11,30]$. It is, however, extremely difficult to measure these quantities at realistic gas pressures in the range of $E / P$ values of engineering interest. Furthermore, although the calculated breakdown voltages are very insensitive to the value 
of $K$, the fact that $K$ is unknown makes it problematic to apply the breakdown criterion in a direct way.

These difficulties can, as shown by Schumann, be circumvented in the following manner. By applying equation (59) to a strictly uniform field of gap length $d$ and pressure $p$ we obtain the following expression

$$
\left(\bar{\alpha}_{0} / p\right) p d=K \text {, }
$$

where $\bar{\alpha}_{0}$ is the effective coefficient of ionization for air which is associated with the uniform-field onset breakdown field strength $E_{0}$ at the gap length $d$, and pressure $p$ reduced to $20{ }^{\circ} \mathrm{C}$. Paschen curve data for atmospheric air are known with sufficient accuracy. These can be expressed in the form $[11,14,31]$

$$
\frac{E_{0}}{p}=B+\frac{C}{\sqrt{p d}}
$$

in which $B$ and $C$ are constants. Equation (61) can be rewritten as

$$
\left[\frac{E_{0}}{p}-B\right]^{2} p d=c^{2}
$$

or as

$$
\frac{K}{c^{2}}\left[\frac{E_{0}}{p}-B\right]^{2} p d=K \text {. }
$$

Thus, from a comparison of (60) and (63) we can deduce that, on the basis of (59) and (61), the functional dependence of $\bar{\alpha} / p$ for atmospheric air can be expressed as

$$
\frac{\bar{\alpha}}{p}=\frac{K}{c^{2}}\left[\frac{E}{p}-B\right]^{2} \text {. }
$$

This is a well established relationship, which was first deduced by Schumann [15]. Experimental studies [11,32] of $\bar{\alpha} / p$ indicate that $K / C^{2}$ is constant, and hence $K$ becomes a constant. The values obtained by Boyd et al. [32] for $B$ and $C$ are

$$
\begin{aligned}
& B=2.42 \mathrm{kV}(\mathrm{mm} \text { bar })^{-1} \\
& C=2.08 \mathrm{kV}(\mathrm{mm} \text { bar })^{-1 / 2 .}
\end{aligned}
$$

Insertion of equation (64) in (59) shows that the criterion for the onset of breakdown in air can be written in the form 


$$
\int_{0}^{z_{0}}[E(z)-p B]^{2} \mathrm{~d} z=p c^{2} .
$$

The unknown constant $K$ is thus replaced by two known constants $B$ and $C$. Since the critical avalanche length $z_{0}$ is the distance from the electrode beyond which a net growth of ionization is impossible, it follows from equation (64) that $z_{0}$ can be obtained from

$$
E\left(z_{0}\right)=p B
$$

unless, of course, the field distribution is such that the avalanche is crossing the gap making $z_{0}=d$.

The field strength $E(z)$ in a divergent field can be written as

$$
E(z)=E(0) f(z) \text {. }
$$

If $f(z)$ is known from a general calculation of the electrostatic field for the entire system, insertion in equations (67) and (68) will yield the onset breakdown field strength at the electrode $E_{0}$.

If the field distribution is not known then the exact values of $f(z)$ will be given by

$$
f(z)=\exp \left[-2 \int_{0}^{z_{0}} H\left(z^{\prime}\right) \mathrm{d} z^{\prime}\right] \text {. }
$$

In this situation the only value of $H(z)$ which is known will be $H(0)$. If, however, the field is known to be divergent everywhere in the region of interest we may assume that the exact value of $H(z)$ will be somewhere between the values of $H(z)$ for the circular cylindrical field and the spherical field, i.e. the values given by equations (54) and (53), respectively. This means that the required onset breakdown field strength $E_{0}$ is to be found in the interval between the values of $E_{0}$ given by these two limiting field configurations for the specified value of the mean curvature $H(0)$ of the electrode surface. These two very simple field situations are therefore of paramount importance.

Since the breakdown onset field strength $E_{0}$ will depend on the mean curvature of the electrode surface it is convenient to introduce a dimensionless curvature factor $\zeta$ defined by 


$$
\zeta=\frac{E_{0}}{p B}, \text { with } \zeta>1,
$$

which means that the breakdown criterion (67) can be written in the form

$$
\int_{0}^{z_{0}}[\zeta f(z)-1]^{2} \mathrm{~d} z=\frac{1}{p}\left(\frac{\mathcal{C}}{B}\right)^{2} .
$$

The critical avalanche length $z_{0}$ is related to $\zeta$ and $f(z)$ by

$$
\zeta f\left(z_{0}\right)=1 .
$$

For the spherical field distribution $f(z)$ is given by

$$
f(z)=\frac{1}{\left(1+H_{0} z\right)^{2}},
$$

where $H_{0}$ is the mean curvature of the electrode surface. Inserting in (72) and integrating leads to the following relation between $p / H_{0}$ and $\zeta$ for the spherical field

$$
p / H_{0}=\frac{3(C / B)^{2}}{\zeta^{2}-6 \zeta+8 \sqrt{\zeta}-3} .
$$

Similarly we obtain for the circular cylindrical field, for which

$$
f(z)=\frac{1}{1+2 H_{0} z},
$$

the following expression

$$
p / H_{0}=\frac{2(C / B)^{2}}{\zeta^{2}-2 \zeta \ln \zeta-1} .
$$

In Figure 1 is shown $\zeta=f\left(p / H_{0}\right)$ calculated from equations (75) and (77). The full line refers to the spherical field and the dashed line to the circular cylindrical field distribution. These two curves can be considered to be the limiting curves for all electrode configurations which are associated with divergent fields. In practice these two curves can be represented by one equation, namely

$$
\zeta=1+2\left[\frac{p / H_{0}}{\text { bar mm }}\right]^{-0.36} \text {. }
$$

It is thus seen that the introduction of the mean curvature in the analysis leads to one formula, which contains the onset breakdown data for air for all possible shapes of electrodes. 


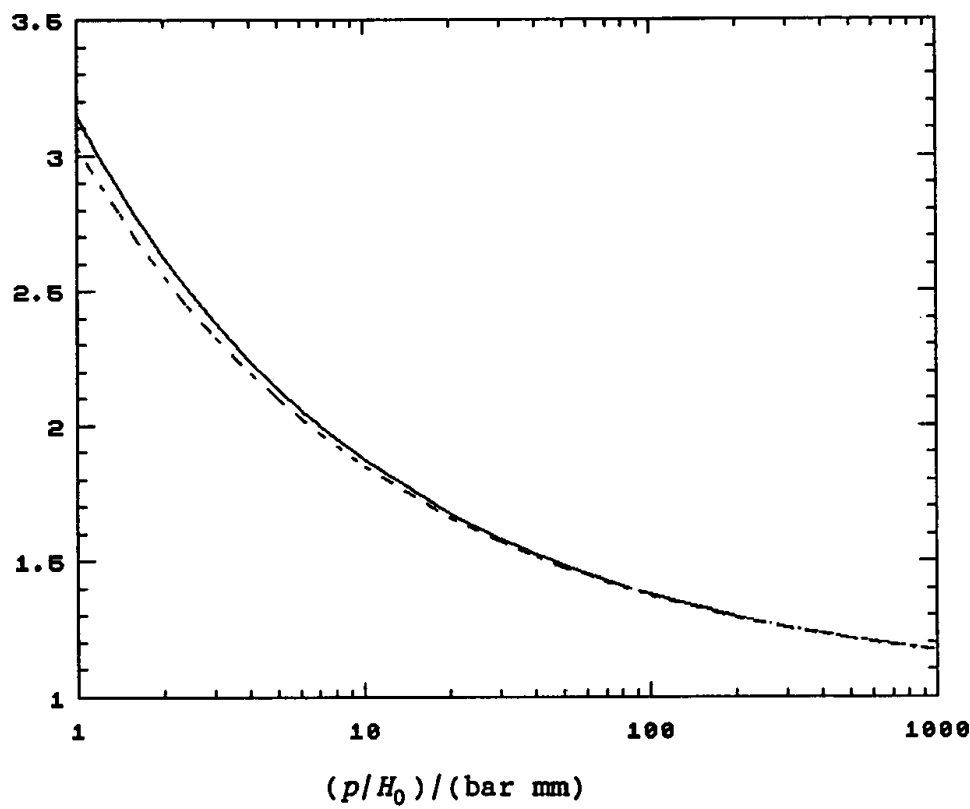

Figure 1. Curvature factor 5 for air. Full line: spherical field distribution.

Dashed line: cylindrical field distribution.

The onset breakdown voltage $U_{0}$ for the electrode arrangement can be calculated from

$$
U_{0}=\eta \zeta B p d \text {, }
$$

in which $\eta$ is the field utilization factor introduced by Schwaiger [33]. It is defined as the ratio between the average field strength of the gap and the maximum field strength at the electrode surface. $B$ is the limiting value of $E / p$ below which growth of tonization is impossible, see equation (65), and $d$ is the gap length. The field utilization factor is known if the field distribution is known. Information about $\eta$ for many standard electrode configurations can be found in the literature, see for example $[28,29,33,34,35,36]$. 
In order to evaluate the effects of approximations in the field distribution it is of interest to consider the variation of the dimensionless quantity $H_{0} z_{0}$ with $p / H_{0}$. These relationships are found from equations (73), (74) and (76). The results are shown in Figure 2 where the full line refers to the spherical field and the dashed line to the circular cylindrical field distribution. It is seen that the approximation given by equation (52) can be used only for relative large electrode dimensions; i.e. $p / H_{0}>100$ bar $\mathrm{mm}$, see also [37].

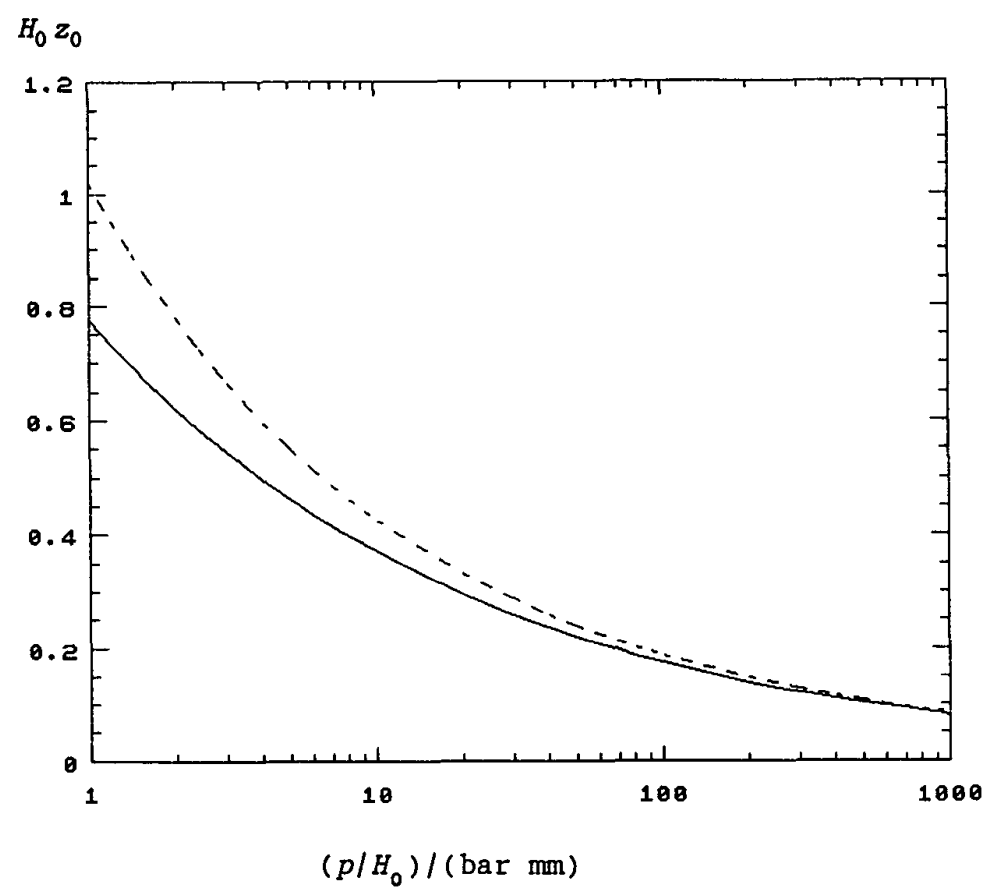

Figure 2. Normalized critical avalanche length $H_{0} z_{0}$ for air.

Full line: spherical field distribution. Dashed line: cylindrical field distribution.

\section{Breakdown in Strongly Electronegative Gases}

In practice the $E / p$ range of interest for any strongly electronegative gas or gas mixture will be around the 
limiting value $(E / p)$ lim, i.e. the value of $E / p$ for which the effective coefficient of ionization $\bar{\alpha}$ is zero. In this range of $E / p$, the magnitude of $\bar{a} / p$ is the difference between two much larger, approximately equal quantities which vary rapidly with $E / P$; viz. those quantities representing the electron production and attachment processes. The variation of $\bar{\alpha}$ with $E$ and $p$ can, consequently, be written in the form

$$
\bar{\alpha}=\bar{\beta} E-\bar{\kappa} p \text {, }
$$

in which $\bar{\beta}$ and $\bar{\kappa}$ are constants.

Measurements of $\bar{\alpha}$ for such gases are very difficult to perform, the reason being that an extremely high degree of field uniformity is required in order to obtain meaningful data [38].

Applying the breakdown criterion to the breakdown of such gases in a strictly uniform field leads to

$$
U_{0}=\frac{K}{\bar{\beta}}+\frac{\bar{K}}{\bar{\beta}} p d \text {. }
$$

This equation can be written in the form

$$
U_{0}=(M+p d)(E / p) \text { Iim }
$$

in which $M=K / \bar{\kappa}$ is the figure of merit for the gas [23]. The part of the Paschen curve which can be linked with the streamer breakdown criterion thus leads to a linear Paschen curve. This linear dependence has been verified experimentally for a large number of electronegative gases. The Paschen curve as a whole is of course nonlinear and exhibits a minimum.

Applications of the streamer criterion to breakdown in nonuniform fields are best performed through the use of Paschen curve data. In many cases this will be the only possible approach simply because of the lack of reliable ionization growth data. Furthermore, the difficulty with the unknown $K$-factor is bypassed through this approach.

Introducing the curvature factor 5 , see equation (71), defined by

$$
\zeta=\left(E_{0} / p\right) /(E / p)_{1 \text { im }} .
$$


where $E_{0}$ is the onset breakdown field strength, the following expressions can be derived from the streamer criterion [23]

with

$$
\int_{0}^{z_{0}}[\zeta f(z)-1] \mathrm{d} z=M / p
$$

$$
\zeta f\left(z_{0}\right)=1
$$

The dimensionless function $f(z)$, see equation (69), describes the variation of the field strength along the field line where the discharge can develop. A knowledge of $(E / p)$ Iim and $M$ is sufficient, therefore, for a calculation of breakdown onset values in any strongly electronegative gas.

The required data can be obtained from measurements in a "uniform-field" gap of the linear part of the Paschen curve. A true uniform field is, however, an idealization. This is not simply because "uniform field" electrodes are difficult to design, the reason is of a fundamental nature. It is namely a logical consequence of the Maxwell theory that a true uniform electric field cannot be established within a volume of finite dimensions. To obtain valid data a low degree of field nonuniformity is a necessary requirement $[39,40]$. If the degree of field nonuniformity associated with "uniform field" gaps is defined as $\left(E_{\mathrm{av}}-E_{\mathrm{min}}\right) / E_{\mathrm{av}}$, where $E_{\mathrm{av}}$ and $E_{\mathrm{min}}$ are the average and minimum field strengths along the gap axis, then valid data are obtained if the following condition is fulfilled

$$
\frac{E_{\mathrm{av}}-E_{\mathrm{min}}}{E_{\mathrm{av}}}<\frac{M}{p d} .
$$

With reference to $\mathrm{SF}_{6}$ this restriction was observed in the work of Boyd and Crichton [41]. Since in the literature reference is often made to the Paschen curve for $\mathrm{SF}_{6}$ adopted by CIGRE [42] it must be emphasized that this Paschen curve is derived from experimental data which do not obey the condition given in equation $(86)$. Valid values for $(E / P)_{1 \text { im }}$ and $M$ cannot, therefore, be obtained from the CIGRE curve.

The basis of equation (86) is that $E_{\text {min }} \geq E_{1 i m}$, such 
that $z_{0}=d$. With the non-fulfillment of this inequality, $z_{0}<d$ and thus the breakdown voltage measurements cannot be analyzed in terms of the Paschen curve. This aspect has been discussed in detail with reference to a recent experimental study [43].

Since the value of $M$ is obtained by extrapolating from the $p d$ interval where the curve is linear, a small uncertainty in the slope of this section, i.e. in $(E / P) 1 \mathrm{im}$, can result in a large uncertainty in the evaluation of $M$. Consequently, to fully exploit the inherent advantages of this method considerable experimental precision is essential. An interesting alternative method by which $M$, but not $(E / p) 1 \mathrm{im}$, can be obtained from measurements in a nonuniform field has been suggested by Qiu and Liu [44].

The use of equations ( 84 ) and ( 85 ) can be illustrated by considering a rotationally symmetric electric field. The field distribution near the surface of the highly stressed electrode will, as a first approximation, be given by equation (55), i.e. $f(z)$ will be given by equation (74). Insertion in equations (84) and (85) yields the following expressions for the curvature factor. $\zeta$ and $H_{0} z_{0}$

$$
\zeta=\left[1+\sqrt{\frac{M}{p / H_{0}}}\right]^{2}
$$

and

$$
H_{0} z_{0}=\sqrt{\frac{M}{p / H_{0}}}
$$

where $H_{0}$ is the mean curvature of the electrode at the axis of the system. In many applications $M \ll p / H_{0}$ and equation ( 87$)$ can then be written in the form

$$
\zeta=1+\sqrt{\frac{4 M}{p / H_{0}}} .
$$

If $M<0.01\left(p / H_{0}\right)$ then $H_{0} z_{0}<0.1$, which means that the simple approximation of the field distribution given in equation (52) may be used.

The figure of merit and $(E / p)_{1 \text { im }}$ for $S_{6}$ are 0.040 bar mm and $8.9 \mathrm{kV}(\mathrm{mm} \text { bar })^{-1}$, respectively. For other strongly electronegative gases $M$ varies with $(E / p) l i m$ as shown in Figure 3. The curve is valid for both unary gases and binary gas mixtures [45]. 


\section{$M /($ bar $\mathrm{mm})$}

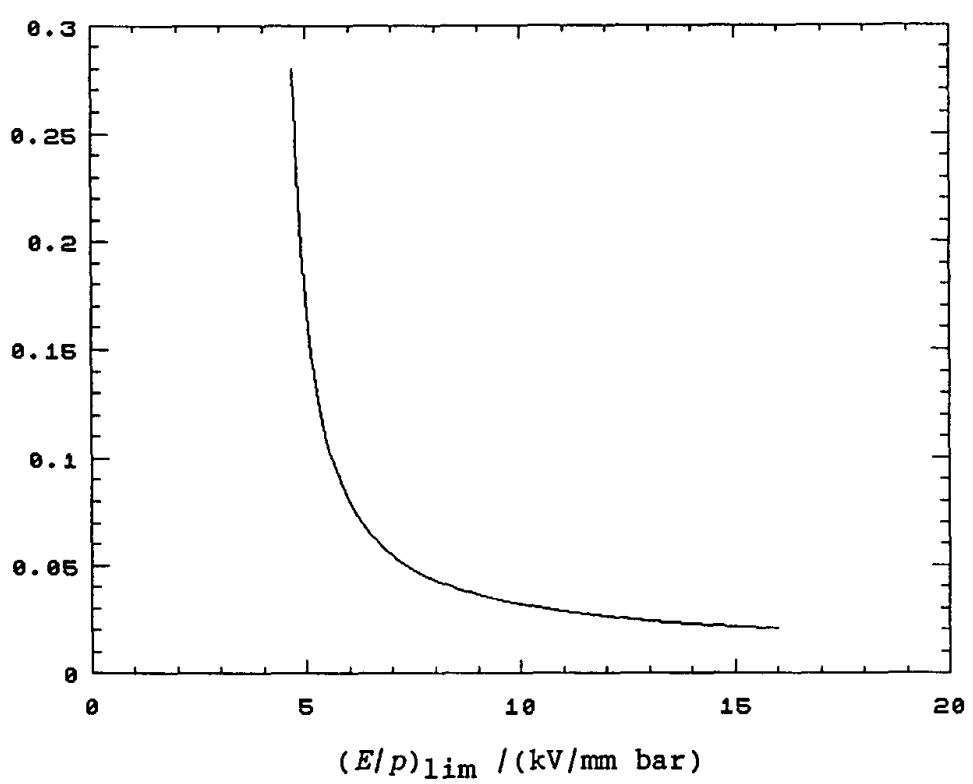

Figure 3. The Figure of Merit $M$ as a function of $(E / P)_{1 \mathrm{im}}$ for strongly-elektronegative gases and gas mixtures.

\section{Surface Roughness and Breakdown}

The successful application of compressed $\mathrm{SF}_{6}$ as a dielectric in gas-insulated substations stimulated a renewed interest in gaseous dielectrics. This lead to a search for gases or gas mixtures with better dielectric properties than $\mathrm{SF}_{6}$. Such gases or gas mixtures will inevitably be strongly electronegative. An important approach to this search is that suggested by Christophorou and his colleagues $[46,47,48]$.

In this molecular approach it is tacitly assumed that a gas for which $(E / P)_{1} \mathrm{im}$ is higher than that for pure $\mathrm{SF}_{6}$ is a better gaseous insulant provided that it can be applied under similar circumstances. From a molecular point of view this is a natural assumption, since no net 
gain of ionization is possible for $E / P$ lower than $(E / p)_{1 i m}$. However, from an engineering point of view $(E / P)$ lim is on its own not a suitable parameter for an assessment of possible design values for the maximum macroscopic $(E / P)$ which can be accepted in practice. This is clearly illustrated by referring to pure $\mathrm{SF}_{6}$. The maximum macroscopic design field strength $E_{\mathrm{d}}$, which can be tolerated in an overvoltage situation in an $\mathrm{SF}_{6}$ insulated GIS without seriously affecting the reliability, is considerably less than that indicated by $(E / P) l i m$ $[49,50]$, i.e.

$$
E_{\mathrm{d}} / p=c(E / p) l \mathrm{im}
$$

in which $c$ is a constant which for compressed $\mathrm{SF}_{6}$ has a value of about 0.5 . The reason for this relatively large difference between the maximum design value and the $1 \mathrm{imi}$ ting value is related to the influence of small microscopic field perturbations caused by the unavoidable presence of electrode surface defects and freely moving particles. The severity of this influence is likely to increase, the more electronegative the gas or gas mixture is relative to $\mathrm{SF}_{6}$.

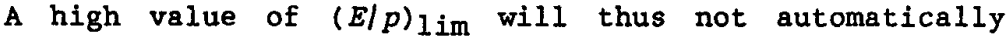
ensure that such a gas could be a suitable alternative to $\mathrm{SF}_{6}$. The $(E / p)$ lim must be supplemented with other data before an assessment can be undertaken of the relevant dielectric properties. The required additional information is the figure of merit $M[51,52]$.

A direct numerical analysis of the effect of surface roughness is not possible since the field distributions associated with actual surface defects are inherently unknown. To circumvent this difficulty, models of defects of various degrees of complexity have been proposed [53, 54]. However, the use of models can lead to specific conclusions which do not have general validity, since on examination these are shown to be associated with properties unique to the model [54].

The only electrostatic field which can be dealt with in a unique way is the macroscopic field distribution. This can be calculated from the idealized geometry of the system, neglecting the effects of all defects. All design parameters must, therefore, refer to this macroscopic field distribution. Hence the parameters for considera- 
tion are the gas pressure $p$ and the design field strength $E_{\mathrm{d}}$, i.e. the maximum macroscopic field strength which can be tolerated under any circumstances in the system.

The true maximum field strength $E_{m}$ which can occur in the system is larger than $E_{\mathrm{d}}$ because of the perturbations of the field due to surface roughness, i.e.

$$
E_{\mathrm{m}}=m E_{\mathrm{d}}, \quad m>1,
$$

where the field enhancement factor $m$ is independent of the selected value of $E_{\mathrm{d}}$. Although $m$ is generally an unknown constant the value of this factor will normally be of the order of 10 or less, see Loeb [56]. Recently, an experimental technique has been developed [57] which allows the $m$ value of a practical surface to be determined. Results confirm that an upper limit of 10 for such surfaces is a reliable measure.

Since the actual field distribution associated with $E_{\mathrm{m}}$ is entirely unknown we cannot calculate the true breakdown strengths of the system. However, breakdown must not occur at $E_{\mathrm{d}}$. We can, therefore, from the streamer breakdown criterion for strongly electronegative gases, see equations (84) and (85), obtain that the following condition must be fulfilled at $E_{\mathrm{d}}$,

$\int_{0}^{z_{0}}$ 0

in which $g(z)$ is the unknown field distribution associated with $E_{\mathrm{m}}$. Although this integral cannot at all be evaluated, it is clearly seen that, the larger $M / p$ is, the larger are the values of the integral which can be tolerated. The larger $M$ becomes the less sensitive is that particular gas to the effect of field disturbances from defects. $M$ can therefore be used as a figure of merit describing this effect $[51,52]$.

The lack of knowledge concerning the $E_{\mathrm{m}}$ field distribution may be circumvented using a comparative technique [23]. If the discharge characteristic for the system in question is known, then this technique allows the direct assessment of a possible replacement gas to be made based solely on this characteristic and the $M$ and $(E / p) l i m$ data of the two gases [58]. 
The real macroscopic breakdown onset field strength $E_{t}$ is called the technical breakdown field strength of the system. When surface roughness influences the breakdown voltage, $E_{t}$ is less than the theoretical breakdown onset field strength $E_{0}$ obtained from the breakdown criterion. This reduction can be described by a surface roughness factor $\xi$ defined by $[49,53]$

$$
E_{\mathrm{t}}=\xi E_{0}, \quad \xi<1 .
$$

Design values for $\xi$ cannot be calculated. These values, which must be fixed on the basis of experience, will depend on the treatment of the conductor surfaces [49, 55]. The technical breakdown voltage $U_{t}$ for the system can be written in the form

$$
U_{\mathrm{t}}=\eta \xi \zeta(E / p)_{1 i m} p d \text {. }
$$

in which $\eta$ is the field utilization factor, i.e. the ratio between the macroscopic average and maximum field strengths related to the gap length $d$, and 5 is the curvature factor which can be obtained from equations (87) or $(89)$.

\section{CONCLUSIONS}

Criteria for the electrical breakdown of gaseous dielectrics are, when expressed in terms of ionization growth parameters, of limited use to high voltage design engineers. In many cases, the lack of fundamental data will make application of these criteria difficult or even impossible. Furthermore, since these will in practice contain at least one parameter which must be chosen arbitrarily, results may be no better than results obtained from rule-of-thumb methods. It is possible, however, to formulate quantitative, nonuniform field, breakdown or onset criteria which are free from such uncertain or unknown parameters.

All the necessary data for the application of these breakdown criteria to a particular gaseous dielectric are obtained directly from the associated Paschen curve, i.e. from uniform field breakdown measurements. No knowledge of the specific values of the lonization and attachment coefficients for the gas is required, 
although a functional dependence $\bar{\alpha}(E)$ is invoked. A distinct advantage accrues since breakdown voltage measurements are much easier to perform than measurements of prebreakdown ionization growth parameters.

It is essential, however, to emphasize that the required Paschen curve data must refer to proper uniform field conditions. The restriction, which is imposed on the acceptable degree of field nonuniformity, has unfortunately not always been observed for the Paschen curve data available in the literature. An example is the Paschen curve for $\mathrm{SF}_{6}$ adopted by CIGRE which does not represent proper uniform field data. The limit for this requirement depends on the gas in question. The more electronegative the gas or gas mixture is, the stricter is the limit to the acceptable degree of field nonuniformity. If this restriction is ignored the compounded error can be far from negligible.

In the present study the emphasis has been placed on the onset of breakdown, i.e. on the lowest possible breakdown voltage. This will normally be synonymous with the d.c. breakdown voltage. Other factors will in practice affect the actual breakdown voltage. These factors are associated with the time lag which is involved in the formation of the breakdown. For atmospheric air this can in special cases be utilized favourably in the design of an apparatus. This aspect is of little relevance in compressed gas systems in which the gaseous dielectric is strongly electronegative e.g. $\mathrm{SF}_{6}$. The adoption of methods which depend on time lag effects cannot in general be recommended since this may lead to a dramatic reduction in the reliability of such a system. Finally, no matter how sophisticated or simple a theory may be, the reliability of the system must remain the dominant feature in all considerations.

\section{ACKNOWLEDGMENT}

The author wishes to thank his colleagues J. Berril, G.C. Crichton, P.W. Karlsson, I.W. McAllister and S. Vibholm for many years of friendly and inspiring cooperation. Moreover the efforts of Kirsten Andersen in preparing the manuscript are highly appreciated. 


\section{REFERENCES}

[1] J.C. Maxwell, A Treatise on Electricity and Magnetism, First Edition, Clarendon Press Oxford, 1873, Vol.1, p. 92.

[2] A. Pedersen, "On the Electrostatics of Probe Measurements of Surface Charge Densities", in L.G. Christophorou and D.W. Bouldin (Eds.), Gaseous Dielectrics V, Pergamon Press New York, 1987, pp. 235-240.

[3] A. Pedersen, "Partial Discharges in Voids in Solid Dielectrics. An Alternative Approach", 1987 Annual Report - Conference on Electrical Insulation and Dielectric Phenomena, IEEE Publication 87CH2462-0, pp. 58-64, 1987.

[4] G.C. Crichton, P.W. Karlsson and A. Pedersen, "On Partial Discharges in Ellipsoidal and Spheroidal Voids", IEEE Trans. Electrical Insulation, Vol.24, pp. 335-342, 1989.

[5] A. von Engel and M. Steenbeck, Elektrische Gasentladungen, Springer-Verlag Berlin, 1932, Vol.1.

[6] W. Shockley, "Currents to Conductors Induced by a Moving Point Charge", J.Appl.Phys., Vol.9, pp. $635-636,1938$.

[7] S. Ramo, "Currents Induced by Electron Motion", Proc. IRE, Vol.27, pp. 584-585, 1939.

[8] B.V. Gnedenko and A.Ya. Khinchin, An Elementary Introduction to the Theory of Probability, Dover Publications New York, 1962.

[9] H. Raether, Electron Avalanches and Breakdown in Gases, Butterworths London, 1964.

[10] J. Berril, J.M. Christensen and A. Pedersen, "Measurement of the Figure of Merit Related to the Effect of Electrode Surface Defects on Breakdown for Strongly Electronegative Gases or Gas Mixtures", Seventh International Conference on Gas Discharges and their Applications, Peter Peregrinus London, 1982, pp. 266-268.

[11] B. Gänger, Der elektrische Durchschlag von Gasen, Springer-Verlag Berlin, 1953.

[12] E. Nasser, Fundamentals of Gaseous Ionization and Plasma Electronics, Wiley-Interscience New York, 1971.

[13] J.M. Meek and J.D. Craggs (Eds.), Electrical Breakdown of Gases, Wiley Chichester, 1978. 
[14] W.0. Schumann, Elektrische Durchbruchfeldstärke von Gasen, Springer-Verlag Berlin, 1923.

[15] W.0. Schumann, "Utber das Minimum der Durchbruchfeldstärke bei Kugelelektroden", Archiv für Elektrotechnik, Vol.12, pp. 593-608, 1923.

[16] J.S. Townsend, Electricity in Gases, Clarendon Press Oxford, 1915.

[17] F. Llewellyn-Jones, Ionization and Breakdown in Gases, Methuen London, 1957.

[18] F. Llewellyn-Jones, Ionization Avalanches and Breakdown, Methuen London, 1967.

[19] International Electrotechnical Commission, Recommendation for Voltage Measurement by means of Sphere-Gaps (One Sphere Earthed), IEC Publication 52 Geneva, 1960

[20] L.B. Loeb and J.M. Meek, The Mechanism of the Electric Spark, Stanford University Press Stanford, 1941.

[21] I.W. McAllister, G.C. Crichton and E. Bregnsbo, "Experimental Study on the Onset of Positive Corona in Atmospheric Air", J.Appl.Phys., Vo1.50, pp. 6797-6805, 1979.

[22] A. Pedersen, "High Voltage Breakdown in Sulfur Hexafluoride", Proc. XVth International Conference on Phenomena in Ionized Gases (Invited Papers), BSSR Academy of Sciences Minsk USSR, $1981, \mathrm{pp}$. 301-310.

[23] A. Pedersen, I.W. McAllister, G.C. Crichton and S. Vibholm, "Formulation of the Streamer Breakdown Criterion and its Application to Strongly Electronegative Gases and Gas Mixtures", Archiv für Elektrotechnik, VoI.67, pp. 395-402, 1984.

[24] K. Rektorys, Survey of Applicable Mathematics, Iliffe Books London, 1969, Chapter 9.

[25] G. Green, An Essay on the Application of Mathematical Analysis to the Theories of Electricity and Magnetism, Nottingham, 1828. Reprinted in Mathematical Papers of the Late George Green ed. N.M. Ferrers, Chelsea New York, 1970.

[26] E. Durand, Electrostatique, Tome II, Problemes Generaux. Conducteurs, Masson Paris, 1966, pp.10-11.

[27] I.W. MCAllister and A. Pedersen, "Green's Differential Equation and Electrostatic Fields", J.Phys.D: Appl.Phys., Vol.21, pp. 1823-1825, 1988 , and Vol.22, p. $473,1989$. 
[28] A. Bouwers, Elektrische Höchstspannungen, Springer-Verlag Berlin, 1939, pp. 126-129.

[29] H. Steinbigler, Anfangsfeldstärken und Ausnutzungsfaktoren rotationssymmetrischer Elektrodenanordnungen in Luft, Dissertation TU Munich, 1969.

[30] H. Hess, Der elektrische Durchschlag in Gasen, Vieweg Braunschweig, 1976.

[31] D.T.A. Blair, "Breakdown Voltage Characteristics", in J.M. Meek and J.D. Craggs (Eds.), Electrical Breakdown of Gases, Wiley Chichester, 1978.

[32] H.A. Boyd, F.M. Bruce and D.J. Tedford, "Sparkover in Long Uniform-Field Gaps", Nature, Vol. 210, pp. 719-720, 1966.

[33] A. Schwaiger, Elektrische Festigkeitslehre, Springer-Verlag Berlin, 1925.

[34] H. Prinz, Hochspannungsfelder, R. Oldenburg Verlag München, Wien, 1969.

[35] D. Kind and H. Kärner, High-Voltage Insulation Technology, Vieweg Braunschweig, 1985.

[36] M. Beyer, W. Boeck, K. Möller and W. Zaengl, Hochspannugstechnik, Springer-Verlag Berlin New York, 1986.

[37] I.W. McAllister and A. Pedersen, "Corona-Onset Field-strength Calculations and the Equivalent Radius Concept", Archiv für Elektrotechnik, Vol. 64, pp. 43-48, 1981 .

[38] A. Pedersen, P.W. Karlsson and J. Lebeda, "The Effect of Field Nonuniformity and Asymmetry on Ionization Current Growth Measurements in Sulfur Hexafluoride", IEEE Trans. Power Apparatus and Systems, Vol. PAS-90, pp. 2175-2180, 1971.

[39] P.W. Karlsson and A. Pedersen, "Inherent Limitations in Uniform Field Discharge Data for $\mathrm{SF}_{6}{ }^{n}$, IEEE Trans. Power Apparatus and Systems, Vol. PAS-91, pp. 1591-1601, 1972.

[40] A. Pedersen, P.W. Karlsson, E. Bregnsbo and T. Munk Nielsen, "Anomalous Breakdown in Uniform Field Gaps in $\mathrm{SF}_{6} "$, IEEE Trans. Power Apparatus and Systems, Vol. PAS-93, pp. 1820-1826, 1974.

[41] H.A. Boyd and G.C. Crichton, "Uniform-Field Breakdown-Voltage Measurements in Sulphur Hexafluoride", Proc. IEE, Vol.119, pp. 275-276, 1972.

[42] G. Luxa, G. Oppermann, J. Vigreux and H. Winkelnkemper, "Paschen Curve for Sulfur Hexafluoride $\left(\mathrm{SF}_{6}\right)^{n}$, Electra, No.32, pp. 75-82, 1974. 
[43] I.W. McAllister and G.C. Crichton, "The Concept of Paschen's Law with Reference to $\mathrm{SF}_{6}{ }^{\prime \prime}$, J.Phys. D: App1.Phys., Vol.20, pp. 1537-1539, 1987.

[44] Y. Qiu and Y.F. Liu, "A New Approach to Measurement of the Figure-of-Merit for Strongly Electronegative Gases and Gas Mixtures", IEEE Trans. Electrical Insulation, Vol. EI-22, pp. 831-834, 1987.

[45] J. Berril, J.M. Christensen and I.W. McAllister, "Measurement of the Figure of Merit $M$ for $\mathrm{CBrClF}_{2}, \mathrm{CBr}_{2} \mathrm{~F}_{2}, \mathrm{CBrF}_{3}$ and $\mathrm{CCIF}_{3}{ }^{n}$, 5th International Symposium on High Voltage Engineering, Vol.1, Paper No. 15.16, Braunschweig, 1987.

[46] L.G. Christophorou, "Elementary Electron-Molecule Interactions and Negative Ion Resonances at Subexcitation Energies and Their Significance in Gaseous Dielectrics", Proceedings 13th International Conference on Phenomena in Ionized Gases: Invited Papers, VEB Export-Import Berlin DDR, 1978, pp. 51-71.

[47] M.O. Pace, L.G. Christophorou, R.D. James, R.Y. Pai, R.A. Mathis and D.W. Bouldin, "Improved Unitary and Multicomponent Gaseous Insulators", IEEE Trans. Electrical Insulation, Vol. EI-13, pp. $31-36,1978$.

[48] L.G. Christophorou, I. Sauers, D.R. James, H. Rodrigo, M.O. Pace, J.G. Carter and S.R. Hunter, "Recent Advances in Gaseous Dielectrics at Oak Ridge National Laboratory", IEEE Trans. Electrical Insulation, Vol. EI-19, pp. 550-566, 1984.

[49] W. Mosch and W. Hauschild, Hochspannungsisolierungen mit Schwefelhexafluorid, VEB Verlag Technik Berlin DDR, 1979.

[50] S. Vibholm and G.C. Crichton, "On the Optimization of Pressure and Surface Roughness Parameters in Compressed $\mathrm{SF}_{6}$ ", Seventh International Conference on Gas Discharges and Their Applications, Peter Peregrinus London, 1982, pp. 259-261.

[51] A. Pedersen, "Evaluation of the Effect of Surface Defects on Breakdown in Strongly Electronegative Gases and Gas Mixtures", in L.G. Christophorou (Ed.), Gaseous Dielectrics II, Pergamon Press New York, 1980, pp. 201-209.

[52] A. Pedersen, "On the Assessment of New Gaseous Dielectrics for GIS", IEEE Trans. Power Apparatus and Systems, Vo1. PAS-104, pp. 2233-2237, 1985. 
[53] A. Pedersen, "The Effect of Surface Roughness on Breakdown in $\mathrm{SF}_{6}$ ", IEEE Trans. Power Apparatus and Systems, Vol. PAS-94, pp. 1749-1754, 1975.

[54] I.W. McAllister, "The Influence of Electrode Macroscopic Curvature upon Surface Roughness Effects in Compressed $\mathrm{SF}_{6}{ }^{\prime}$, Archiv für Elektrotechnik, Vol.62, pp. 43-49, 1980.

[55] I.W. McAllister, "The Concept of Electrode Surface Roughness with Reference to Discharge Phenomena in Strongly Electronegative Gases", IEEE Trans. Electrical Insulation, Vol. EI-21, pp. 659-662, 1986.

[56] L.B. Loeb, Fundamental Processes of Electrical Discharges in Gases, John Wiley New York, 1939, pp. 473-474.

[57] I.W. McAllister and G.C. Crichton, "An Experimental Method to Determine the Electrostatic Field Enhancement Factor of a Practical Conductor Surface", IEEE Trans. Electrical Insulation, Vol.24, pp. 325-333, 1989.

[58] G.C. Crichton and S. Vibholm, "Discharge Onset Voltage Prediction for a Gas-Insulated System Via the Figure-of-Merit Concept", IEEE Trans. Electrical Insulation, Vol.EI-22, pp. 819-823, 1987. 